\title{
Application of Diffusion Tensor Imaging in Obstructive Nephropathy
}

\author{
Hua-Jian Xu ${ }^{1}$, Hanwen Zhang $\mathbb{C D}^{1}{ }^{1,}$, Juan Yu ${ }^{1}$, Fan Lin $^{1}$ and Yi Lei ${ }^{1}$ \\ ${ }^{1}$ Department of Radiology, the First Affiliated Hospital of Shenzhen University, Health Science Center, Shenzhen Second People's Hospital, 3002 SunGangXi Road, Shenzhen, \\ China \\ "Corresponding author: Department of Radiology, the First Affiliated Hospital of Shenzhen University, Health Science Center, Shenzhen Second People's Hospital, 3002 \\ SunGangXi Road, Shenzhen, China. Email: zhwstarcraft@outlook.com
}

Received 2021 March 30; Revised 2021 August 02; Accepted 2021 August 15.

\begin{abstract}
Background: Obstructive nephropathy is a common clinical disease.

Objectives: To explore the value of diffusion tensor imaging (DTI) in obstructive nephropathy.

Materials and Methods: Forty healthy Sprague-Dawley (SD) rats were examined in this study. Thirty-two animals underwent complete obstruction of the left ureter, while eight animals underwent a sham surgery. Magnetic resonance imaging (MRI) was performed before surgery and within different intervals after surgery. Eight rats from the experimental group and two rats from the sham group were used in each interval. Following MRI, the animals were sacrificed and sent for medical examinations. The scanning sequences included positioning, transverse T2-weighted (T2W), coronal, and coronal DTI sequences. Image postprocessing was performed after DTI to measure DTI parameters, including apparent diffusion coefficient (ADC) and fractional anisotropy (FA), and to reconstruct DTI fiber traces. One-way analysis of variance was used to compare the parameters between the cortex and medulla and between different intervals.

Results: The fiber tracing showed that the obstructed renal fiber bundles were sparse and disordered. The ADC and FA values of the renal cortex, extrarenal medulla, and inner medulla decreased with prolonged hydrops and were negatively correlated with the expression of alpha-smooth muscle actin $(\alpha$-SMA) and the renal tubulointerstitial lesion grade $(\mathrm{r}<0, \mathrm{P}<0.001)$. Comparison of the cortex, extrarenal medulla, and inner medulla showed the following trends for the ADC and FA values: cortex $>$ extrarenal medulla $>$ inner medulla and cortex $<$ extrarenal medulla $<$ inner medulla, respectively.

Conclusions: DTI in obstructive nephropathy not only can reflect the degree of renal interstitial fibrosis and accurately indicate the renal function, but also can provide information regarding renal blood perfusion, water metabolism, and ultrastructural changes.
\end{abstract}

Keywords: Obstructive Nephropathy, Diffusion Tensor Imaging, Animal Experiment

\section{Background}

Obstructive nephropathy is a renal disease, caused by impaired urinary circulation in the urinary tract, which produces back pressure and affects the normal physiology of the renal parenchyma (1). Hydronephrosis and renal atrophy, caused by ureteral obstruction, are the most common diseases in urology, with an incidence rate as high as $5 \%$. Overall, understanding the occurrence and pathogenic mechanism of obstructive nephropathy is essential for the clinical selection of a reasonable time for surgery and monitoring the prognosis postoperatively (2).

Following ureteral obstruction, the pathophysiology, anatomical morphology, and hemodynamics of the kidneys will change, depending on the duration and degree of obstruction. The early stage of acute obstruction mainly manifests as renal tubular lesions. With prolonged obstruction, renal tubular epithelial cells undergo balloon- ing, atrophy, degeneration, necrosis, and tubulointerstitial fibrosis, which can ultimately lead to renal atrophy, functional impairment, and damage. Generally, tubulointerstitial fibrosis plays a key role in this process. It is currently believed that tubulointerstitial fibrosis is related to interactions between the extracellular matrix (ECM), cytokines, cells, and growth factors $(3,4)$.

Transforming growth factor- $\beta 1$ (TGF- $\beta 1$ ) is recognized as the most critical cytokine in interstitial fibrosis (5). Besides, a variety of interstitial cells are involved in the occurrence and development of renal interstitial fibrosis. Studies have shown that mesenchymal cells and renal tubular epithelial cells may undergo phenotypic changes, express alpha-smooth muscle actin ( $\alpha$-SMA), and exhibit certain characteristics of smooth muscle cells, including increased proliferation, expression of ECM components, and secretion.

The $\alpha$-SMA-positive cells play a key role in the progres- 
sion of renal disease to end-stage renal failure (6). In 2007, Liu et al. reported a regular increase in the $\alpha$-SMA expression as an important indicator of renal interstitial fibrosis following obstructive hydronephrosis (7). Meanwhile, the renal blood flow gradually decreases with prolonged obstruction. In a previous study, the renal blood flow decreased to $50 \%$ compared to the control group at 24 hours after complete ureteral obstruction and further decreased to $30 \%$ after six days, $20 \%$ after two weeks, and $12 \%$ after eight weeks (8).

Doppler ultrasound, radionuclide scintigraphy, computed tomography (CT), and excretory cystourethrography are often used clinically to examine ureteral obstruction. However, the information that can be obtained by these modalities is limited and cannot provide microscale data, such as renal pathophysiology or hemodynamics $(9,10)$. In recent years, researchers have concentrated on the development of non-invasive and safe functional magnetic resonance imaging (fMRI) techniques, which do not require the use of contrast agents, such as diffusionweighted imaging (DWI), diffusion tensor imaging (DTI), arterial spin-labeled fMRI (ASL-fMRI), and blood-oxygenlevel-dependent fMRI (BOLD-fMRI), to reflect pathological changes and blood perfusion of the kidneys at microscopic levels (11-13). Moreover, some scholars have done early explorations of DTI in obstructive nephropathy; however, the results are not pathologically confirmed, and the level of evidence is low $(14,15)$.

\section{Objectives}

This study aimed to apply DTI in obstructive nephropathy and to examine its correlation with pathology by analyzing the correlations of apparent diffusion coefficient (ADC) and fractional anisotropy (FA) of the obstructed renal cortex and medulla with the degree of renal tubular interstitial damage and the expression of $\alpha$-SMA as a fibrosisrelated factor (16). Besides, the relationships between the DTI parameter map, fiber bundle tracing map, and renal ultrastructure were investigated.

\section{Materials and Methods}

\subsection{Experimental Animals}

Forty-four healthy Sprague-Dawley (SD) rats were purchased from the Animal Center of Guangzhou University of Traditional Chinese Medicine (license number: SCXK 20130200). Two of the animals died accidentally due to anesthesia, and two others had severely deformed images due to motion artifacts; these animals were removed from the study. Overall, 19 female and 21 male rats, weighing $252.6 \pm$
$14.5 \mathrm{~g}$, were examined in this study. The average age of the animals was two months.

First, preoperative MRI was performed for each animal. Thirty-two rats underwent surgery, while eight rats underwent a sham surgery. MRI was performed at one, three, five, and seven days postoperatively. Eight animals in the experimental group and two animals in the sham group were scanned in each time interval. The ear-piercing method was used to mark the animals. Immediately after the scan, the animals were sacrificed and sent for medical examinations. Chloral hydrate (10\%) was injected intraperitoneally at a dose of $3.3 \mathrm{~mL} / 100 \mathrm{~g}$ body weight for anesthesia.

This study was approved by the Ethics Committee of Jinan University, Guangzhou, China. The experimental process was performed according to the ethical guidelines for animal research.

\subsection{Animal Model}

Ligation with a No. 1 silk thread was used to establish a model of complete irreducible unilateral ureteral obstruction. In this method (Figure $1 \mathrm{~A}$ ), the rat was anesthetized with an intraperitoneal injection of $10 \%$ chloral hydrate (3.3 $\mathrm{mL} / 100 \mathrm{~g})$; the skin was prepared and routinely disinfected with iodophor twice. A median abdominal incision was made, and the skin was cut to expose the kidney. The left ureter was found near the iliac blood vessel and freed at approximately $1.0 \mathrm{~cm}$ from the junction of the renal pelvis and ureter. Next, the ureter was ligated with a No. 1 silk thread and ligated again at $0.5 \mathrm{~cm}$ distal from the first site of ligation. The abdominal cavity was then sutured layer by layer with No. 4 threads, and 40,000 U of penicillin was injected intramuscularly for three days.

Moreover, in the sham group, the abdomen was cut open after anesthesia induction, and only the left ureter was freed; then, the abdominal wall was sutured layer by layer. All surgeries were performed aseptically throughout the procedure, and 0.2 to $0.3 \mathrm{~mL}$ of chloral hydrate was administered to maintain anesthesia as needed.

\subsection{Assessment Method}

\subsubsection{Preparation and Equipment}

All rats were refused food for 12 hours and water for four hours before MRI. A GE Healthcare 3.0T Discovery MR750 superconducting scanner (Chicago, IL, USA) was used with an HD wrist array upper coil and a postprocessing workstation [Sun Advantage Workstation (ADW) 4.5]. For scanning, the animal was placed in a supine position, with the limbs fixed and the head advanced. 


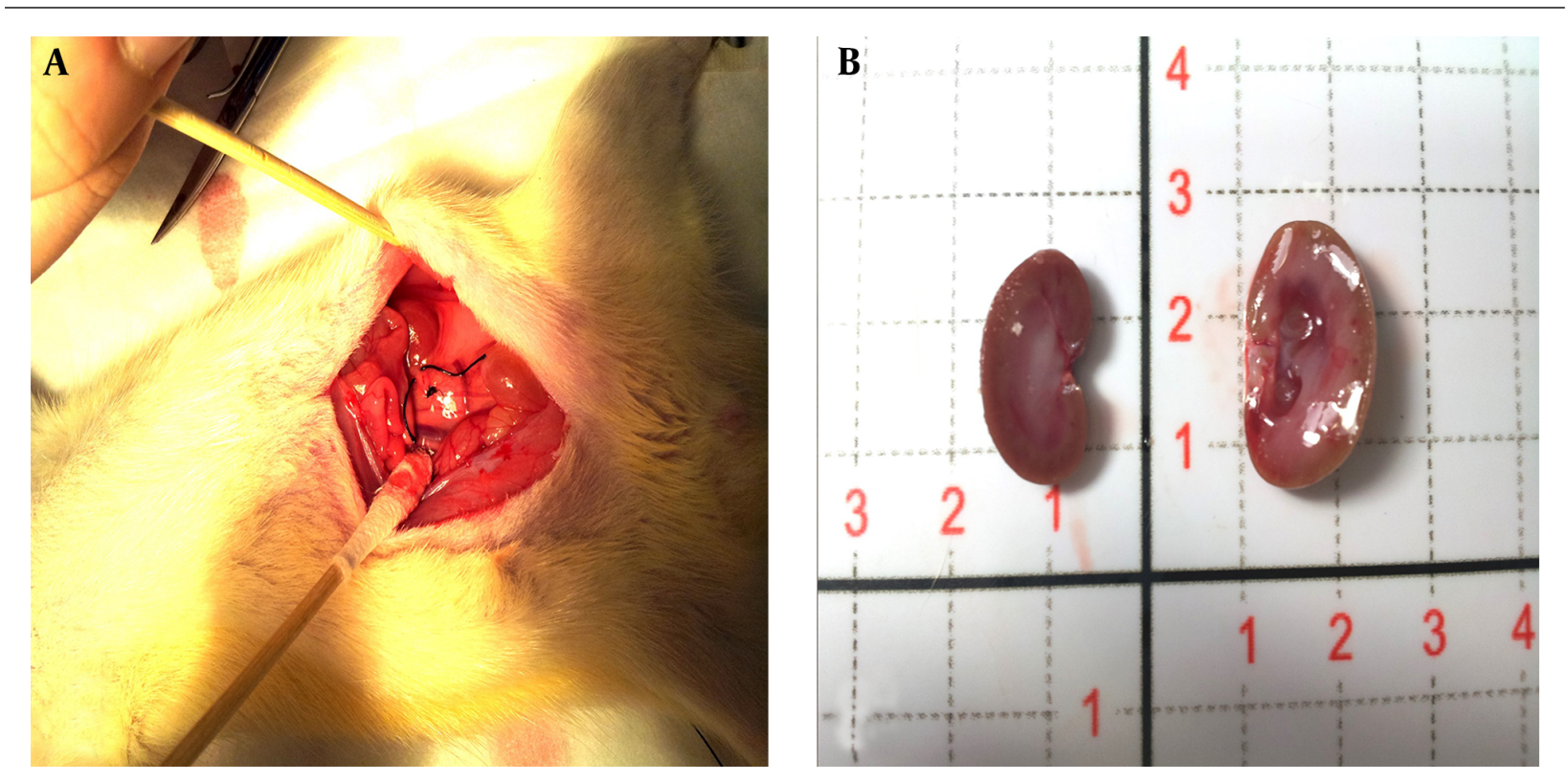

Figure 1. A, The surgical model. The ureter is freed at approximately $1.0 \mathrm{~cm}$ from the junction of the renal pelvis with the ureter and ligated with a No. 1 silk thread; the ureter is ligated again $0.5 \mathrm{~cm}$ distal to the first ligation site. B, A sectional view of the renal hilum of the kidneys (the left kidney is the hydronephrotic kidney). Three days after obstruction, the left renal pelvis is significantly dilated (Juan, Y. Application of intravoxel incoherent motion imaging and diffusion-tensor imaging in obstructive nephropathy. [Master] Jinan University, Guangzhou, Guangdong China, 2014).

\subsubsection{Scanning Sequences and Parameters}

The scanning sequences and parameters were as follows:

- Positioning sequence: A three-plane positioning single-shot fast spin echo (FSEloc) sequence was acquired with the following parameters: (1) repetition time (TR), 7.2 ms; (2) echo time (TE), $2.4 \mathrm{~ms}$; (3) layer thickness, $3.0 \mathrm{~mm}$; (4) interval, $1.0 \mathrm{~mm}$; (5) field of view (FOV), $12 \mathrm{~cm} \times 12 \mathrm{~cm}$; (6) matrix, $256 \times 192$; (7) number of excitations (NEX), 1.0; and (8) scan time, $21 \mathrm{sec}$ (two positioning scans were acquired).

- Transverse T2 weighted image (T2WI) sequence: A fast relaxation-fast spin echo (FR-FSE)-XL/90 sequence was acquired with the following parameters: (1) TR, $1405 \mathrm{~ms}$; (2) TE, $81 \mathrm{~ms}$; (3) layer thickness, $2.0 \mathrm{~mm}$; (4) interval, $0.2 \mathrm{~mm}$; (5) FOV, $8 \times 5.6 \mathrm{~cm}$; (6) bandwidth, $20.8 \mathrm{kHz}$; (7) matrix, 320 $\times$ 256; (8) NEX, 2.0; and (9) scan time, $73 \mathrm{sec}$.

- Coronal T2WI sequence: An FRFSE-XL/90 sequence was acquired with the following parameters: (1) TR, $1500 \mathrm{~ms}$; (2) TE, $79.1 \mathrm{~ms}$; (3) layer thickness, $3.0 \mathrm{~mm}$; (4) layer interval, $0.6 \mathrm{~mm}$; (5) FOV, $10 \times 7 \mathrm{~cm}$; (6) bandwidth, $20.8 \mathrm{kHz}$; (7) matrix, $320 \times 256$; (8) NEX, 4.0; and (9) scan time, 75 sec.

- Coronal DTI sequence: A spin echo (SE)/echo planar imaging (EPI) sequence was acquired with the following parameters: (1) b-value, 500;(2)TR, $3000 \mathrm{~ms}$; (3) TE, $75.4 \mathrm{~ms}$; (4) layer thickness, $3.0 \mathrm{~mm}$; (5) layer interval, $0.6 \mathrm{~mm}$; (6) FOV, $10.0 \times 5 \mathrm{~cm}$; (7) bandwidth, $167 \mathrm{kHz}$; (8) matrix size, 96 $\times$ 64; (9) NEX, 2.0; (10) diffusion-sensitive gradient direction, 6; and (11) acquisition time, 2 min and 39 sec.

\subsubsection{Image Postprocessing Analysis}

The FuncTool of GE ADW 4.5 Workstation was used for image postprocessing and analysis. The raw intravoxel incoherent motion (IVIM) and DTI data were transferred to the workstation to obtain maps of DTI parameters (ADC and FA). The regions of interest (ROIs) were placed on the upper, middle, and lower poles of the renal cortex, extrarenal medulla, and inner medulla at the coronal hilar level, avoiding blood vessels and areas of renal pelvic dilation and calyces. All ROIs had exactly the same size $(1 \sim 2$ $\mathrm{mm}^{2}$ ) and included at least five pixels. Fiber tracking was also used by considering the renal parenchyma as the target site to reconstruct the renal parenchymal diffusion tensor traces and analyze the position and shape of renal fiber bundles.

\subsection{Specimen Collection and Analysis}

\subsubsection{Collection and Processing of Specimens}

MRI was performed at one, three, five, and seven days postoperatively. The animals were then sacrificed, and their kidneys were removed, cut in half at the hilar level, and soaked in $4 \%$ neutral formalin solution for 24 hours at room temperature. Next, the sections were dehydrated, embedded in paraffin, and sectioned ( $3 \mu \mathrm{m}$ ) (Figure $1 \mathrm{~B})$. 


\subsubsection{Staining Method}

The surface of the renal hilum was cut as much as possible to examine lesions. Hematoxylin and eosin ( $\mathrm{H} \&$ E) staining and immunohistochemical staining were performed, using the streptavidin-alkaline phosphatase (SP) method for the latter. The primary antibody was against $\alpha$ SMA, and the secondary antibody was a PIKA universal EnVision secondary antibody. The kits were purchased from Jitai Yuancheng Biological Co., Ltd. (Santa Cruz Biotechnology, Inc., Dallas, TX, USA).

\subsubsection{Analysis of Immunohistochemical Results and Renal} Tubulointerstitial Lesions

Two senior pathologists observed the results of cell staining by microscopy and used known positive sections as positive controls to quantitatively analyze the level of $\alpha$ SMA expression, based on the intensity, range, and distribution of immunohistochemical staining. Tubulointerstitial renal fibrotic lesions involved inflammatory cell infiltration, renal tubular atrophy, and interstitial fibrosis. Besides, pathological sections were observed under light microscopy after H\&E staining, and renal tubulointerstitial lesions were semi-quantitatively graded according to the method reported by Mezzano et al. (17).

\subsection{Statistical Analysis}

Statistical analysis was performed in SPSS 19.0 (Chicago, Illinois, USA). Variables were compared between the groups and between the renal cortex and medulla using one-way analysis of variance. Dunnett's test was also used to compare variables between the experimental and sham groups preoperatively and postoperatively $(1,3,5$, and 7 days). Moreover, the groups and parameters were compared in the cortex, extrarenal medulla, and inner medulla in each group by the Student-Newman-Keuls (SNK) method.

Besides, a line graph of changes in the parameters of the cortex, extrarenal medulla, and inner medulla with prolonged obstruction was plotted. Also, the correlation of each parameter with the expression of $\alpha$-SMA and the severity of renal tubulointerstitial lesions was analyzed by Pearson's correlation coefficient test. Besides, the correlation of $\alpha$-SMA expression with the severity of renal tubulointerstitial lesions and the duration of hydrops was analyzed by Spearman's rank correlation coefficient test. Data were considered statistically significant at $\mathrm{P}<0.05$.

\section{Results}

\subsection{MRI Results}

The T2WI and DTI sequences of the rat kidneys in the sham group clearly showed stratification of renal cortex, extrarenal medulla, and inner medulla, no dilation of renal pelvis, and differences in the ADC and FA values between the cortex and medulla on pseudocolor images. The DTI fiber trace map could clearly show the performance of the rat obstruction model in different stages (Figure 2).

\subsection{Statistical Analysis of DTI Parameters}

\subsubsection{ADC Analysis}

Tables 1- 3 show changes in the ADC of the renal cortex, inner medulla, and extrarenal medulla in the obstructed and healthy groups, besides differences between the cortex and medulla. There was no significant difference in the ADC values between the experimental and sham groups preoperatively. One day after surgery, the ADC of the extrarenal medulla was lower in the experimental group compared to the sham group $(\mathrm{P}=0.005)$. On the third day, the ADC values of the cortex, extrarenal medulla, and inner medulla were lower in the experimental group compared to the control group $(\mathrm{P}=0.001, \mathrm{P}<0.001$, and $\mathrm{P}<0.001$, respectively).

On the fifth and seventh days postoperatively, the ADC values of the cortex, extrarenal medulla, and inner medulla were lower in the experimental group compared to the control group $(\mathrm{P}<0.001)$. Comparison of the cortex and medulla regarding ADC in each group showed the following trend, with a statistically significant difference $(\mathrm{P}<0.05)$ : cortex $>$ extrarenal medulla $>$ inner medulla (Table 3). Moreover, according to Table 2, the ADC values of the cortex and medulla in the contralateral kidneys of the experimental group were not significantly different from those of the sham group $(\mathrm{P}>0.05)$. Besides, there was a significant difference in the ADC values between the cortex and medulla in each group $(\mathrm{P}<0.05)$.

\subsubsection{FA Analysis}

Tables 4 - 6 show changes in the FA of the renal cortex, inner medulla, and extrarenal medulla in the obstructed and contralateral kidneys, as well as differences between the cortex and medulla. As shown in Table 4, there was no significant difference in terms of ADC between the experimental and sham groups preoperatively. One day after renal obstruction, the FA of the cortex was lower in the experimental group compared to the sham group; however, the difference was not statistically significant $(\mathrm{P}=0.312)$. Also, the FA of the outer and inner medulla was lower in the experimental group compared to the control group ( $\mathrm{P}$ $=0.005$ and $\mathrm{P}=0.003$, respectively) .

On the third postoperative day, the FA of the cortex, extrarenal medulla, and inner medulla was lower in the experimental group compared to the control group ( $\mathrm{P}=$ $0.004, \mathrm{P}<0.001$, and $\mathrm{P}<0.001$, respectively). On the fifth 


\begin{tabular}{|c|c|c|c|c|c|}
\hline Variables & Sham group & First postoperative day & Third postoperative day & Fifth postoperative day & Seventh postoperative day \\
\hline Cortex & $2.03 \pm 0.11$ & $1.89 \pm 0.07$ & $1.75 \pm 0.07$ & $1.62 \pm 0.06$ & $1.53 \pm 0.09$ \\
\hline P-value & - & 0.103 & 0.001 & $<0.001$ & $<0.001$ \\
\hline Extrarenal medulla & $1.83 \pm 0.06$ & $1.72 \pm 0.04$ & $1.58 \pm 0.05$ & $1.47 \pm 0.06$ & $1.36 \pm 0.06$ \\
\hline P-value & - & 0.005 & $<0.001$ & $<0.001$ & $<0.001$ \\
\hline Inner medulla & $1.63 \pm 0.06$ & $1.60 \pm 0.06$ & $1.43 \pm 0.10$ & $1.26 \pm 0.06$ & $1.08 \pm 0.04$ \\
\hline P-value & - & 1.000 & $<0.001$ & $<0.001$ & $<0.001$ \\
\hline
\end{tabular}

Abbreviation: ADC, apparent diffusion coefficient.

${ }^{\text {a }}$ P-values are the results of pairwise comparisons between the experimental and sham groups at different time points.

${ }^{\mathrm{b}}$ P-value $<0.05$ is statistically significant ( $\mathrm{n}=8$ per group).

\begin{tabular}{|c|c|c|c|c|c|}
\hline Variables & Sham group & First postoperative day & Third postoperative day & Fifth postoperative day & Seventh postoperative day \\
\hline Cortex & $1.97 \pm 0.12$ & $2.09 \pm 0.18$ & $2.05 \pm 0.13$ & $2.02 \pm 0.08$ & $2.10 \pm 0.11$ \\
\hline P-value & - & 0.747 & 1.000 & 1.000 & 0.457 \\
\hline Extrarenal medulla & $1.74 \pm 0.13$ & $1.78 \pm 0.10$ & $1.78 \pm 0.06$ & $1.77 \pm 0.09$ & $1.83 \pm 0.13$ \\
\hline P-value & - & 1.000 & 1.000 & 1.000 & 0.946 \\
\hline Inner medulla & $1.63 \pm 0.15$ & $1.70 \pm 0.17$ & $1.57 \pm 0.11$ & $1.61 \pm 0.15$ & $1.74 \pm 0.22$ \\
\hline P-value & - & 1.000 & 1.000 & 1.000 & 1.000 \\
\hline
\end{tabular}

Abbreviation: ADC, apparent diffusion coefficient.

${ }^{a} \mathrm{P}$-values are the results of pairwise comparisons between the experimental and sham groups.

${ }^{\mathrm{b}} \mathrm{P}<0.05$ is statistically significant $(\mathrm{n}=8$ per group).

\begin{tabular}{|c|c|c|c|}
\hline \multirow{2}{*}{ Variables } & \multicolumn{3}{|c|}{ ADC; P-value } \\
\hline & Cortex vs. extrarenal medulla & Cortex vs. inner medulla & Extrarenal medulla vs. inner medulla \\
\hline Sham group & 0.003 & $<0.001$ & $<0.001$ \\
\hline First postoperative day & $<0.001$ & $<0.001$ & 0.001 \\
\hline Third postoperative day & 0.001 & $<0.001$ & 0.002 \\
\hline Fifth postoperative day & $<0.001$ & $<0.001$ & $<0.001$ \\
\hline Seventh postoperative day & $<0.001$ & $<0.001$ & $<0.001$ \\
\hline
\end{tabular}

Abbreviation: ADC, apparent diffusion coefficient.

${ }^{a} \mathrm{P}<0.05$ is considered statistically significant.

\begin{tabular}{|c|c|c|c|c|c|}
\hline Variables & Sham group & First postoperative day & Third postoperative day & Fifth postoperative day & Seventh postoperative day \\
\hline Cortex & $1.01 \pm 0.13$ & $0.83 \pm 0.16$ & $0.70 \pm 0.13$ & $0.69 \pm 0.14$ & $0.57 \pm 0.22$ \\
\hline P-value & - & 0.312 & 0.004 & 0.002 & $<0.001$ \\
\hline Extrarenal medulla & $2.56 \pm 0.37$ & $1.96 \pm 0.33$ & $1.50 \pm 0.24$ & $1.12 \pm 0.10$ & $0.93 \pm 0.22$ \\
\hline P-value & - & 0.005 & $<0.001$ & $<0.001$ & $<0.001$ \\
\hline Inner medulla & $3.37 \pm 0.65$ & $2.89 \pm 0.44$ & $2.42 \pm 0.34$ & $2.16 \pm 0.21$ & $1.81 \pm 0.33$ \\
\hline P-value & - & 0.003 & $<0.001$ & $<0.001$ & $<0.001$ \\
\hline
\end{tabular}

Abbreviation: FA, fractional anisotropy.

${ }^{a} \mathrm{P}$-value is determined by pairwise comparisons between the experimental and sham groups at different time points.

${ }^{\mathrm{b}} \mathrm{P}<0.05$ is statistically significant ( $\mathrm{n}=8$ per group). 


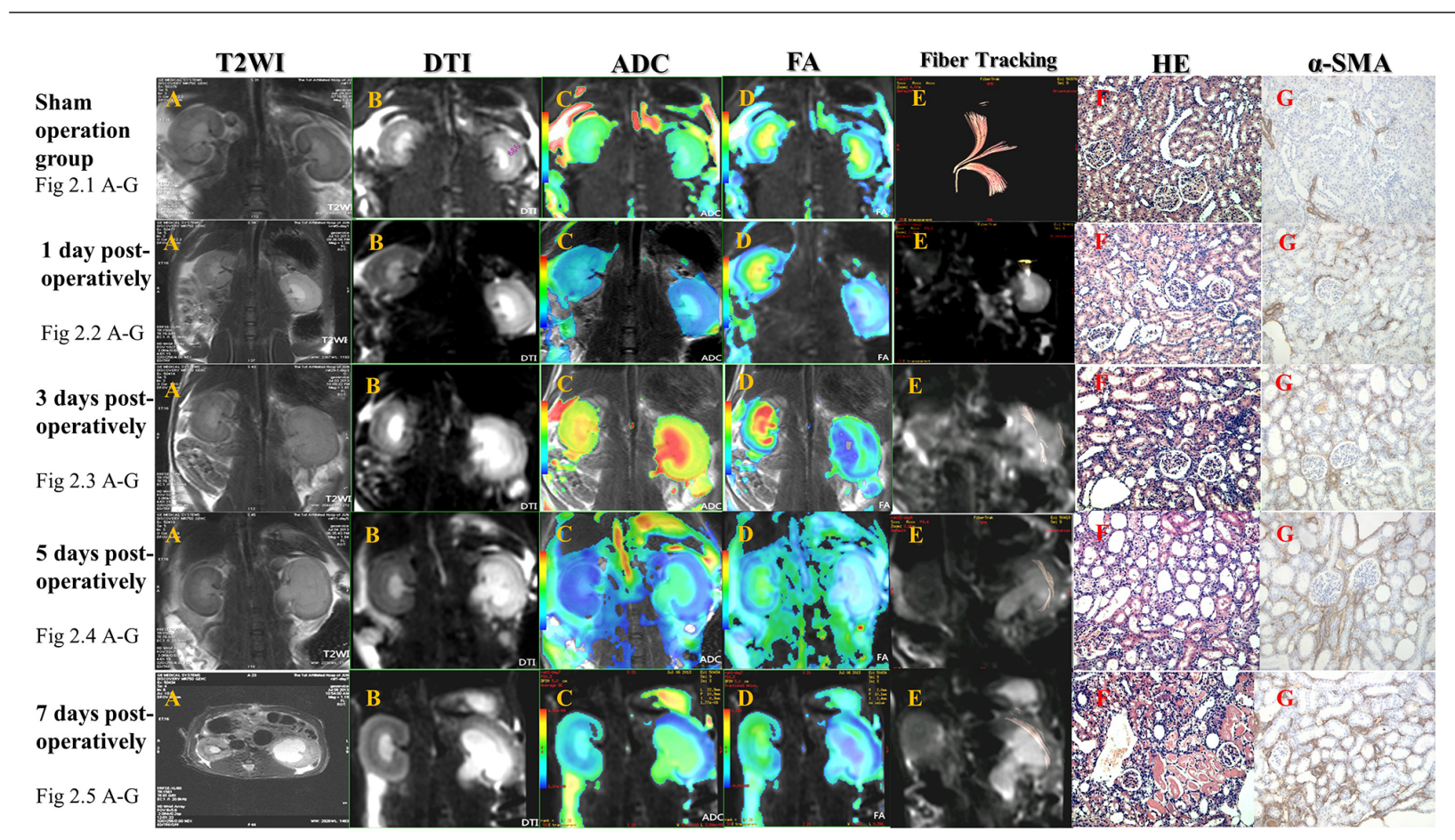

Figure 2. DTI fiber tracing shows that in a normal kidney, fibers diverge from the renal hilum at the center and are arranged regularly (fig 2.1). One day after renal obstruction in rats, the renal pelvis is slightly dilated. T2WI shows the inner medullary edema, and coronal DTI shows an increased signal in the inner medulla, with distinct signals in the cortex, extrarenal medulla, and inner medulla. Pseudocolor images represent the outlines and changes in renal regions more precisely. The DTI fiber trace map only shows traces for some fiber bundles from the renal hilum to the cortex (fig 2.2). Three days after renal obstruction, the outline is slightly enlarged, as well as the renal pelvis. T2WI shows that the inner medullary edema is aggravated, without involving the extrarenal medulla. Coronal DTI shows that the inner medullary signal is increased, and the cortical, extrarenal medullary, and inner medullary layers are clear. Pseudocolor images better represent the outlines of the kidneys. The DTI fiber trace map shows traces of some fiber bundles from the renal hilum to the cortex; the fiber arrangement is disordered, and there are some irregular fiber bundles (fig 2.3). Five days after obstruction, the outline of the kidney is enlarged, and the renal pelvis is dilated. T2WI shows edema in the renal cortex and medulla. DTI indicates increased signals in the cortex and medulla; the cortex, extrarenal medulla, and inner medulla are clearly shown. Pseudocolor images better represent the outlines of the kidneys. The DTI fiber trace map shows no traces of normal radial fiber bundles, and the fiber bundles are arranged irregularly (fig 2.4). Seven days after obstruction, the contour of the kidneys is significantly enlarged, and the renal pelvis is severely expanded. T2WI shows further aggravation of the renal parenchymal edema with the renal cortex involvement. Coronal DTI shows increased signals from the renal cortex and medulla, while the cortex, extrarenal medulla, and inner medulla are blurred. Pseudocolor images better represent the outlines of the kidneys. The DTI fiber trace map shows no traces of normal radial fiber bundles, and the fiber bundles are arranged irregularly (fig 2.5). Abbreviations: DTI, diffusion tensor imaging; FA, fractional anisotropy; ADC, apparent diffusion coefficient; $\alpha$-SMA, alpha-smooth muscle actin; T2WI, T2 weighted image; HE, hematoxylin and eosin.

\begin{tabular}{|c|c|c|c|c|c|}
\hline Variables & Sham group & First postoperative day & Third postoperative day & Fifth postoperative day & Seventh postoperative day \\
\hline Cortex & $1.39 \pm 0.31$ & $1.39 \pm 0.44$ & $1.47 \pm 0.70$ & $1.21 \pm 0.06$ & $1.31 \pm 0.21$ \\
\hline P-value & - & 0.783 & 0.892 & 0.980 & 0.754 \\
\hline Extrarenal medulla & $2.27 \pm 0.45$ & $2.27 \pm 0.76$ & $2.45 \pm 0.50$ & $2.33 \pm 0.39$ & $2.55 \pm 0.64$ \\
\hline P-value & - & 1.000 & 1.000 & 1.000 & 1.000 \\
\hline Inner medulla & $3.63 \pm 0.68$ & $3.80 \pm 0.34$ & $4.00 \pm 0.41$ & $3.97 \pm 3.66$ & $3.78 \pm 0.54$ \\
\hline P-value & - & 1.000 & 0.972 & 0.988 & 1.000 \\
\hline
\end{tabular}

Abbreviation: FA, fractional anisotropy.

${ }^{\text {a }}$ P-value is determined by pairwise comparisons between the experimental group and the sham group at different time points.

${ }^{\mathrm{b}} \mathrm{P}<0.05$ is statistically significant $(\mathrm{n}=8$ per group).

postoperative day, the FA of the cortex, extrarenal medulla, and inner medulla was lower in the experimental group compared to the control group $(\mathrm{P}=0.002, \mathrm{P}<0.001$, and $\mathrm{P}<0.001$, respectively). On the seventh postoperative day, the FA of the cortex, extrarenal medulla, and inner medulla was lower in the experimental group compared to the control group $(\mathrm{P}<0.001)$. Based on the comparison of the cortex and medulla, the FA showed the following trend in 


\begin{tabular}{|c|c|c|c|}
\hline \multirow{2}{*}{ Variables } & \multicolumn{3}{|c|}{ FA; P-value } \\
\hline & Cortex vs. extrarenal medulla & Cortex vs. inner medulla & Extrarenal medulla vs. inner medulla \\
\hline Sham group & $<0.001$ & $<0.001$ & 0.003 \\
\hline First postoperative day & $<0.001$ & $<0.001$ & $<0.001$ \\
\hline Third postoperative day & $<0.001$ & $<0.001$ & $<0.001$ \\
\hline Fifth postoperative day & $<0.001$ & $<0.001$ & $<0.001$ \\
\hline Seventh postoperative day & 0.031 & $<0.001$ & $<0.001$ \\
\hline
\end{tabular}

Abbreviation: FA, fractional anisotropy.

${ }^{a} \mathrm{P}<0.05$ is considered statistically significant.

each group: cortex $<$ extrarenal medulla $<$ inner medulla. A significant difference was found between the groups ( $P$ $<0.05$ ) (Table 6). According to Table 5, the FA of the cortex and medulla of the contralateral kidney was not significantly different between the experimental and sham groups ( $P>0.05)$, while the FA of the cortex and medulla was significantly different in all groups $(\mathrm{P}<0.05)$.

\subsection{Pathological Findings}

Table 7 shows the expression of $\alpha$-SMA and the grade of renal tubulointerstitial lesions on the obstructed side in each group; the data presented in this table were analyzed by Spearman's rank correlation test. The correlation coefficient between the expression of $\alpha$-SMA and the duration of obstruction was $0.869(\mathrm{P}<0.001)$. The results showed that the expression of $\alpha$-SMA increased with the prolongation of hydronephrosis and increasing severity of renal tubulointerstitial lesions. The correlation coefficient between the grade of renal tubulointerstitial lesions and duration of obstruction was $0.924(\mathrm{P}<0.001)$. In other words, the severity of renal tubulointerstitial lesions increased with the prolongation of water accumulation, especially on the third day.

4.4. Correlation Analysis of DTI Parameters, $\alpha$-SMA Expression, and Renal Tubulointerstitial Lesion Grade in Obstructive Nephropathy

\subsubsection{Apparent Diffusion Coefficient}

With the prolongation of hydronephrosis, the degree of hydronephrosis increased, and the ADC of the external renal medulla showed the fastest change, as it started to decrease on the first day after obstruction. The renal cortex and intrarenal medulla also started to decrease on the third postoperative day. The ADC of the renal cortex, extrarenal medulla, and inner medulla showed strong negative correlations with the expression of $\alpha$-SMA and the grade of renal tubulointerstitial lesions $(\mathrm{P}<0.001)$ (Figure $3 A)$.

\subsubsection{Fractional Anisotropy}

After obstruction, the FA of the renal cortex, extrarenal medulla, and inner medulla decreased with the prolongation of hydronephrosis and increased severity of hydronephrosis. There was a strong negative correlation between FA and both the expression of $\alpha$-SMA and the grade of renal tubulointerstitial lesions $(\mathrm{P}<0.001)$ (Figure $3 \mathrm{~B})$.

\section{Discussion}

In this study, coronal DTI clearly showed stratification of the renal parenchyma in the SD rats, including the cortex, extrarenal medulla (outer and inner bands), and inner medulla, while these changes were more clear on coronal T2WI (13). With the prolongation of hydronephrosis, the renal cortex and medulla signals gradually increased in T2WI and DTI, and changes in the inner medulla were observed the earliest. The first day after obstruction, the boundary between the cortex, extrarenal medulla, and inner medulla became gradually blurred, but could be still distinguished.

The pseudocolor images could display the outlines of the kidneys and differences in color between the layers more precisely. The layer boundaries were most obvious on the ADC and FA maps, which conforms to the results of the statistical analysis of ADC and FA (18). The DTI fiber trace map clearly showed that in a normal kidney, the fibers diverge from the renal hilum at the center and are arranged regularly, while after the induction of hydronephrosis, the fibers are arranged in a disordered manner. The present results showed that DTI is superior to other sequences in representing the anatomical structures of the kidneys and is a suitable technique for studying the anatomy of the kidneys; this finding is consistent with the results of previous studies $(15,19)$.

The pathomorphological study of obstructive hydronephrosis in rats showed that the early stage of acute obstruction mainly manifests as renal tubular lesions. With a prolonged obstruction, the amount of renal tubulointerstitial fibrotic tissue increased, leading to renal 


\begin{tabular}{|c|c|c|c|c|c|c|c|c|c|c|}
\hline \multirow{2}{*}{ Groups } & \multicolumn{5}{|c|}{$\alpha$-SMA expression } & \multicolumn{5}{|c|}{ Tubulointerstitial lesion grade } \\
\hline & - & $\mathbf{1 +}$ & $2+$ & $3+$ & Total & Grade 0 & Grade I & Grade II & Grade III & Total \\
\hline Sham group & 8 & & & & 8 & 8 & & & & 8 \\
\hline First postoperative day & 4 & 3 & 1 & & 8 & 5 & 3 & & & 8 \\
\hline Third postoperative day & & 4 & 2 & 2 & 8 & & 3 & 5 & & 8 \\
\hline Fifth postoperative day & & 1 & 2 & 5 & 8 & & & 3 & 5 & 8 \\
\hline Seventh postoperative day & & & 1 & 7 & 8 & & & 1 & 7 & 8 \\
\hline Total & 12 & 8 & 6 & 14 & 40 & 13 & 6 & 9 & 12 & 40 \\
\hline
\end{tabular}

Abbreviation: $\alpha$-SMA, alpha-smooth muscle actin.
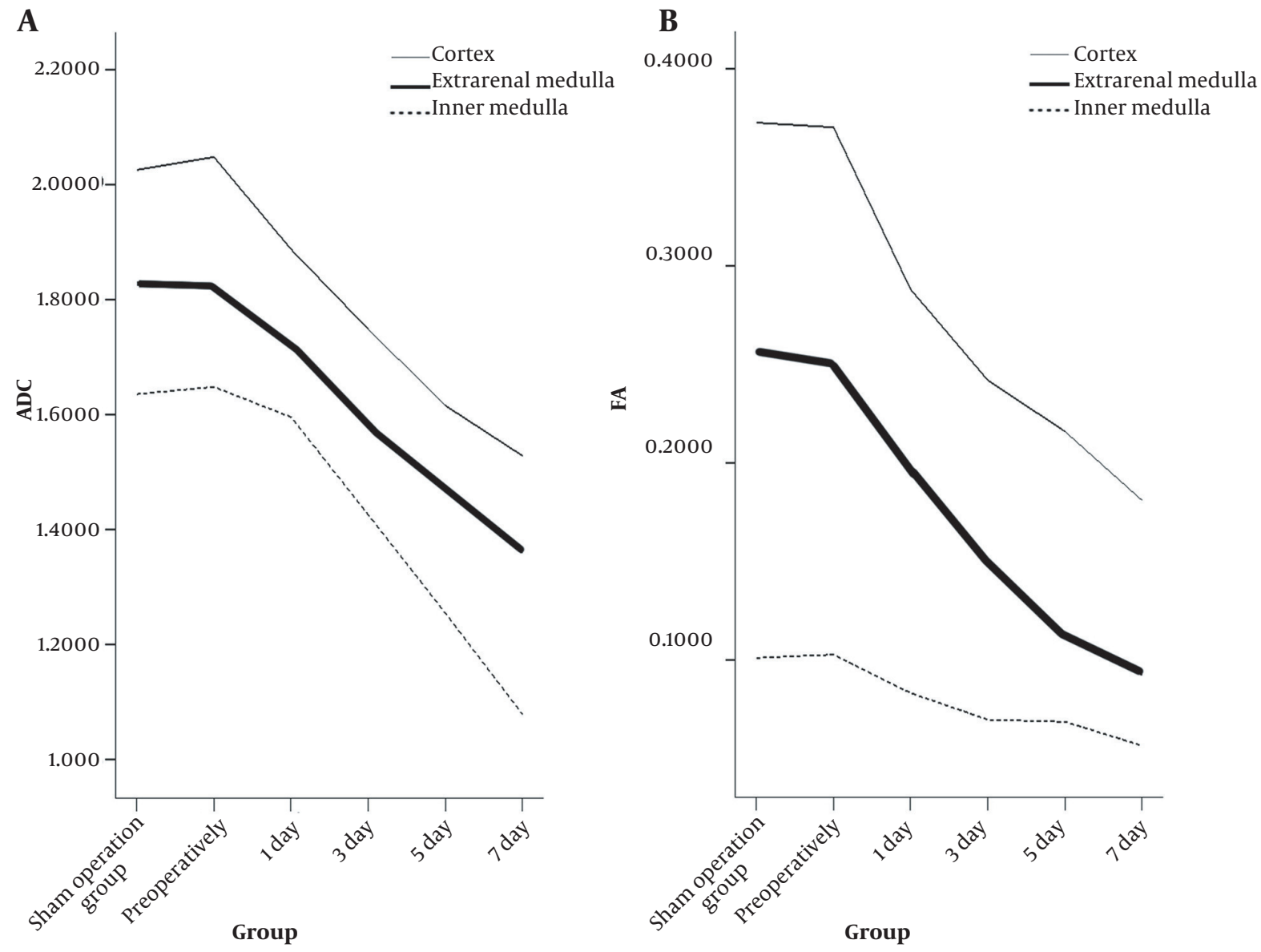

Figure 3. A, The apparent diffusion coefficient (ADC) trend in the cortex, extrarenal medulla, and inner medulla in each group; B, The fractional anisotropy (FA) trend in the cortex, extrarenal medulla, and inner medulla in each group.

atrophy and renal dysfunction. In this study, one day after obstruction, there were no obvious changes in the glomerulus or renal pelvis. Renal tubules were slightly dilated, some renal tubular epithelial cells were swollen, a small amount of scattered inflammatory cells infiltrated into the renal interstitium, and a small amount of fibrous tissue proliferation was detected; the interstitial lesion grade was + . 
Three days after obstruction, H\&E staining showed glomerular cell edema, renal tubule dilation, epithelial cell edema and necrosis, extensive inflammatory cell infiltration, and few fibers in the tubulointerstitium; the lesion grade was ++ . Five days after obstruction, H\&E staining showed glomerular cell edema and necrosis, some interstitial red blood cells, clearly dilated renal tubules, some tubular atrophy and collapse, tubular epithelial cell atrophy and necrosis, and extensive inflammatory cell infiltration and fibrosis in the tubulointerstitium; the lesion grade was +++.

Seven days after obstruction, H\&E staining showed glomerular atrophy, mild fibrosis, further tubular expansion and collapse, tubular epithelial cell atrophy and necrosis, and extensive inflammation in the tubulointerstitium; the lesion grade was +++. The results showed that fibrosis began to appear in the renal interstitium three days after acute complete obstruction. In line with previous research, this study indicated the specific presentation time of the earliest signs of fibrosis in an acute unilateral upper ureteral obstruction model with hydronephrosis.

In the present study, the ADC and FA values showed significant differences between the renal cortex, extrarenal medulla, and inner medulla. The ADC showed a decreasing trend of cortex $>$ extrarenal medulla $>$ inner medulla, while the FA values showed the opposite trend. The ADCs reported in previous studies are inconsistent with our results. In this regard, Muller and Namimoto, by adopting a small b-value, found that the ADC of the medulla was greater than that of the cortex $(20,21)$. They believed that the water content of the medulla was much greater than that of the cortex and that the degree of dispersion might be higher.

It is possible that previous studies failed to distinguish the effects of diffusion and perfusion, resulting in differences in the ADCs between the cortex and medulla. Similarly, in the present study, DTI was performed with a bvalue of $500 \mathrm{~s} / \mathrm{mm}^{2}$, which included the effects of both diffusion and perfusion, and comparison of ADCs between the cortex and medulla was of little significance (22). The FA in most previous studies was higher in the medulla compared to the cortex, reflecting a difference in the ultrastructure of the renal medulla and cortex. The renal medulla is mainly composed of renal tubules, collecting ducts, and a small number of interstitial and small nutrient-supplying blood vessels. Overall, water molecules diffuse more rapidly in the kidneys along the long axis of the tubule; therefore, the FA of the renal medulla is significantly higher than that of the renal cortex, and the directionality of the medulla is clearer.

In the present study, after the induction of obstructive nephropathy, the ADC and FA of the cortex, extrarenal medulla, and inner medulla decreased significantly due to a series of pathophysiological changes in the kidneys. The ADC and FA were closely related to the expression of $\alpha$-SMA and the grade of renal tubulointerstitial lesions. The higher the expression of $\alpha$-SMA and the grade of renal tubulointerstitial lesions were, the lower the ADC and FA values would be; this finding suggested a negative correlation between these variables. Therefore, with the aggravation of renal tubulointerstitial fibrosis, the ADC and FA values gradually decrease. On the fiber trace map, the fibers in the normal kidneys were distributed radially from the renal hilum to the renal cortex, with a regular shape. However, the structure of the renal parenchyma changed after the accumulation of water, and the shape of fibers became irregular (23).

The results of this study showed that the DTI technology not only can be used to quantify differences in the ultra-microanatomical structure of the kidneys (greater directionality of the medulla than the cortex), but also can reflect early morphological changes in the renal function through fiber tracing. Moreover, on DTI for obstructive nephropathy, the ADC and FA showed significant negative correlations with the expression of $\alpha$-SMA and the severity of renal tubulointerstitial lesions; in other words, as the degree of renal interstitial fibrosis increased, the ADC and FA values both progressively decreased.

In conclusion, DTI parameters in obstructive nephropathy not only can provide physiological and anatomical information regarding the microscopic aspects of renal function, but also can indirectly reflect the degree of renal fibrosis, which is helpful for evaluating renal function, guiding clinical treatment, and monitoring prognosis.

\section{Footnotes}

Authors' Contribution: Study conception and design, $\mathrm{H}$ W.Z. and H-J.X.; Analysis and interpretation of data, J.Y. and F.L.; Drafting of the manuscript, Y.L.; Critical revision of the manuscript for important intellectual content, H-W.Z., F.L., and J.Y.; and Statistical analysis, H-J.X. The authors would like to thank Ms. Siling Gu for her assistance in this research.

Conflict of Interests: The authors declare no conflict of interest.

Ethical Approval: This experiment was approved by the Ethics Committee of Jinan University, and the experimental process was performed according to the animal ethics guidelines. A total of 44 healthy SD rats were purchased from the Animal Center of Guangzhou University of Traditional Chinese Medicine with the license number, SCXK 
2013-0200.

Funding/Support: This study was supported by a grant from the Basic Plan Program of Shenzhen, China (No. JCYJ20180228163333734). Lin Fan (MD, PhD) received the grant.

\section{References}

1. Ichii O, Otsuka S, Namiki Y, Hashimoto Y, Kon Y. Molecular pathology of murine ureteritis causing obstructive uropathy with hydronephrosis. PLoS One. 2011;6(11). e27783. doi: 10.1371/journal.pone.0027783. [PubMed: 22114694]. [PubMed Central: PMC3218045].

2. Cenic A, Nabavi DG, Craen RA, Gelb AW, Lee TY. A CT method to measure hemodynamics in brain tumors: Validation and application of cerebral blood flow maps. AJNR Am J Neuroradiol. 2000;21(3):462-70. [PubMed: 10730636]. [PubMed Central: PMC8174983].

3. Chiou YY, Chiu NT, Wang ST, Cheng HL, Tang MJ. Factors associated with the outcomes of children with unilateral ureteropelvic junction obstruction. J Urol. 2004;171(1):397-402. doi: 10.1097/01.ju.0000101381.32320.78. [PubMed: 14665942].

4. Zeisberg M, Maeshima Y, Mosterman B, Kalluri R. Renal fibrosis. Extracellular matrix microenvironment regulates migratory behavior of activated tubular epithelial cells. Am J Pathol. 2002;160(6):20018. doi: 10.1016/So002-9440(10)61150-9. [PubMed: 12057905]. [PubMed Central: PMC1850832].

5. Sager C, Lopez JC, Duran V, Burek C, Perazzo E. Transforming growth factor-beta1 in congenital ureteropelvic junction obstruction: Diagnosis and follow-up. Int Braz J Urol. 2009;35(3):315-25. doi: 10.1590/s1677-55382009000300008. [PubMed: 19538767].

6. Chebotareva NV, Bobkova IN, Varshavskii VA, Golitsyna EP, Kozlovskaia LV. [The role of smooth muscle alpha-actin in development of renal fibrosis in patients with chronic glomerulonephritis]. Ter Arkh. 2006;78(5):17-21. Russian. [PubMed: 16889044].

7. Liu YM, Zhang Y, He LQ. [Experimental study on effect of Kangxianling on rat renal interstitial fibrosis]. Zhongguo Zhong Xi Yi Jie He Za Zhi. 2007;27(10):901-4. Chinese. [PubMed: 17990457].

8. Vaughan ED, Sorenson EJ, Gillenwater JY. The renal hemodynamic response to chronic unilateral complete ureteral occlusion. Invest Urol. 1970;8(1):78-90. [PubMed: 5433182].

9. Green MA, Hutchins GD. Positron emission tomography (PET) assessment of renal perfusion. Semin Nephrol. 2011;31(3):291-9. doi: 10.1016/j.semnephrol.2011.05.008. [PubMed: 21784278].

10. Murphy GPJC. Urologic cancer. Cancer. 2015;62:1800-7. doi: 10.1002/1097-0142(19881015)62:1.

11. English PJ, Testa HJ, Lawson RS, Carroll RN, Edwards EC. Modified method of diuresis renography for the assessment of equivocal pelviureteric junction obstruction. Br J Urol. 1987;59(1):10-4. doi: 10.1111/j.1464-410x.1987.tb04570.x. [PubMed: 3828681].
12. Chu WC, Lam WW, Chan KW, Yeung CK, Lee KH, Sihoe JD. Dynamic gadolinium-enhanced magnetic resonance urography for assessing drainage in dilated pelvicalyceal systems with moderate renal function: preliminary results and comparison with diuresis renography. BJU Int. 2004;93(6):830-4. doi: 10.1111/j.1464-410X.2003.04725.x. [PubMed: 15049999].

13. Seif M, Mani LY, Lu H, Boesch C, Reyes M, Vogt B, et al. Diffusion tensor imaging of the human kidney: Does image registration permit scanning without respiratory triggering? J Magn Reson Imaging. 2016;44(2):327-34. doi:10.1002/jmri.25176. [PubMed: 26871263].

14. Nery F, Szczepankiewicz F, Kerkela L, Hall MG, Kaden E, Gordon I, et al. In vivo demonstration of microscopic anisotropy in the human kidney using multidimensional diffusion MRI. Magn Reson Med. 2019;82(6):2160-8. doi: 10.1002/mrm.27869. [PubMed: 31243814]. [PubMed Central: PMC6988820].

15. Ries M, Jones RA, Basseau F, Moonen CT, Grenier N. Diffusion tensor MRI of the human kidney. J Magn Reson Imaging. 2001;14(1):42-9. doi: 10.1002/jmri.1149. [PubMed: 11436213].

16. Notohamiprodjo M, Glaser C, Herrmann KA, Dietrich O, Attenberger UI, Reiser MF, et al. Diffusion tensor imaging of the kidney with parallel imaging: Initial clinical experience. Invest Radiol. 2008;43(10):67785. doi:10.1097/RLI.ob013e31817d14e6. [PubMed:18791409].

17. Mezzano SA, Droguett MA, Burgos ME, Ardiles LG, Aros CA, Caorsi I, et al. Overexpression of chemokines, fibrogenic cytokines, and myofibroblasts in human membranous nephropathy. Kidney Int. 2000;57(1):147-58. doi: 10.1046/j.1523-1755.2000.00830.x. [PubMed: 10620196].

18. Cutajar M, Clayden JD, Clark CA, Gordon I. Test-retest reliability and repeatability of renal diffusion tensor MRI in healthy subjects. Eur J Radiol. 2011;80(3):e263-8. doi: 10.1016/j.ejrad.2010.12.018. [PubMed: 21227619].

19. Zhou JY, Wang YC, Zeng CH, Ju SH. Renal functional MRI and its application. J Magn Reson Imaging. 2018;48(4):863-81. doi: 10.1002/jmri.26180. [PubMed: 30102436].

20. Muller MF, Prasad PV, Bimmler D, Kaiser A, Edelman RR. Functional imaging of the kidney by means of measurement of the apparent diffusion coefficient. Radiology. 1994;193(3):711-5. doi: 10.1148/radiology.193.3.7972811. [PubMed: 7972811].

21. Namimoto T, Yamashita Y, Mitsuzaki K, Nakayama Y, Tang Y, Takahashi M. Measurement of the apparent diffusion coefficient in diffuse renal disease by diffusion-weighted echo-planar MR imaging. J Magn Reson Imaging. 1999;9(6):832-7. doi: 10.1002/(sici)15222586(199906)9:6<832::aid-jmri10>3.0.co;2-1. [PubMed: 10373031].

22. Gurses B, Kilickesmez O, Tasdelen N, Firat Z, Gurmen N. Diffusion tensor imaging of the kidney at 3 Tesla MRI: Normative values and repeatability of measurements in healthy volunteers. Diagn Interv Radiol. 2011;17(4):317-22. doi: 10.4261/1305-3825.DIR.3892-10.1. [PubMed: 21108183].

23. Gaudiano C, Clementi V, Busato F, Corcioni B, Ferramosca E, Mandreoli $\mathrm{M}$, et al. Renal diffusion tensor imaging: Is it possible to define the tubular pathway? A case report. Magn Reson Imaging. 2011;29(7):1030-3. doi: 10.1016/j.mri.2011.02.032. [PubMed: 21616621]. 